\title{
Stellungnahme der FMH zum Artikel von Virgile Woringer
}

\author{
Christine Romanna, Hanspeter Kuhnb \\ a Dr. med., Mitglied des FMH-Zentralvorstandes, Departementsverantwortliche Gesundheitsförderung und Prävention; \\ ${ }^{b}$ Fürsprecher, Leiter Abteilung Rechtsdienst FMH
}

Virgile Woringer setzt sich aus der Perspektive des schulärztlichen Dienstes einer grossen Schweizer Stadt für eine ärztliche Meldepflicht im Zusammenhang mit Kindesschutzfragen ein. Er erachtet die Waadtländer Meldepflicht im Zusammenhang mit Kindesschutzfragen als sozusagen natürlich («Ce devoir particulier de signaler parait assez naturel, dans la mesure où la société doit pouvoir compter avec le concours de ceux qui sont particulièrement aptes à la détection des mauvais traitements en raison de leur position et de leurs compétences professionnelles») und kritisiert die Stellungnahme des FMH-Zentralvorstandes vom 18. März 2014. Interessanterweise vermutet er hinter dem Einsatz der FMH für ein Melderecht statt einer Meldepflicht einen primär deutschschweizerischen Standpunkt ( ... le poids de la majorité de mes confrères alémaniques») - sonst heisst es oft, das Arztgeheimnis werde in der Romandie besser verteidigt als in der Deutschschweiz. Sein Beitrag geht davon aus, dass mehr Meldungen per se gut sind, und er erwähnt weder die Risiken der Meldepflicht noch die Literatur zum Thema.

Dies waren die zentralen Punkte der FMH-Stellungnahme: «Die FMH begrüsst ein gesetzliches Melderecht, das die heute je nach Kanton unterschiedliche Rechtslage im Kindesschutz einheitlich regelt. Begrüsst wird insbesondere die Ausdehnung des Melderechts auf Gefährdungssituationen, d.h. bevor eine strafbare Handlung vorgefallen ist.

Abzusehen ist hingegen von einer Meldepflicht, weil diese sich oft kontraproduktiv auswirken kann. Eine Meldepflicht führt zu einer Aushöhlung des Berufsgeheimnisses und damit zur Untergrabung jenes Vertrauensverhältnisses, das für die Ausübung des Berufs und für die niederschwellige Zugänglichkeit des Behandlungsangebotes für die Patienten unabdingbar ist. Die Chancen und Risiken einer Meldung und der beste Meldezeitpunkt müssen sorgfältig abgewogen werden. Dabei spielen auch Faktoren wie Verfahrensdauer und Beweisfragen eine Rolle (steht am Ende Aussage gegen Aussage und droht eine Re-Traumatisierung des Kindes?). Wichtig ist auch eine niederschwellige Zusammenarbeit der Fachper- sonen, die beruflich mit Kindern arbeiten, mit den spezialisierten Opferhilfestellen.»

Die FMH stützte ihre Stellungnahme wesentlich auf die fundierte und auch aus heutiger Sicht lesenswerte Analyse der Stiftung Kinderschutz Schweiz ab (Vernehmlassungsantwort vom 10. März 2014 zur Änderung des Schweizerischen Zivilgesetzbuches (Kindesschutz), http://kinderschutz.ch/cmsn/ de). Diese hält unter anderem fest:

- «Eine Interessenabwägung im Einzelfall ist Voraussetzung für diesen Entscheid und rückt in den Mittelpunkt der Entscheidungsfindung. Indem diese Personen nicht der Meldepflicht unterstehen, wird das für die Ausübung der betroffenen Berufe wichtige Vertrauensverhältnis geschützt. Die Erleichterung des Melderechts kann die Meldebereitschaft bei dieser Gruppe von Fachpersonen erhöhen. [...]

- Eine Meldung an die KESB muss mit Sorgfalt und Professionalität angegangen werden, damit sie zielführend ist und weder zu früh noch zu spät kommt. Eine zu spät erfolgte Meldung kann verheerende Folgen für das Kind haben. Eine überstürzte und unüberlegte Meldung entspricht ebenfalls nicht immer dem Kindeswohl, beispielsweise wenn das Vertrauensverhältnis zwischen meldender Person und Klientin oder Klient gefährdet ist. Bevor ein gewisser Schweregrad der Gefährdung erreicht ist, ist der Schutz eines möglicherweise gefährdeten Kindes oft nur unter freiwilliger Kooperation der Eltern möglich. Eine allgemeine Meldepflicht und damit verbundenes vorschnelles Vorgehen, kann aus verschiedenen Gründen kontraproduktiv sein. Es muss mit einer Untergrabung des Vertrauensverhältnisses gerechnet werden. Erfolgschancen und Auswirkungen eines straf- oder zivilrechtlichen Verfahrens können zu wenig bedacht werden. Eine Fachperson muss in der Lage sein, diese Abwägungen anzustellen und über den Zeitpunkt der Meldung zu entscheiden.»

Aus diesen Gründen setzt sich die FMH für Melderechte beim Kindesschutz ein, lehnt hingegen Meldepflichten $\mathrm{ab}$. 